\title{
Empirical Analysis of Use and Gratifications to Government Affairs of new media
}

\author{
Jinli Jia \\ Communication College. Zhengzhou Normal University, Zhengzhou, 450044, China \\ houyin69@163.com
}

Keywords: Government Affairs Microblog; Government Affairs WeChat; Use and Gratifications

\begin{abstract}
Government Affairs Microblog and WeChat are the main carriers of government new media. Based on the theory of "use and gratifications", this paper conducts statistical research on the use of government affairs microblog and WeChat, to analysis the basic situation of these new media and the satisfaction condition of public's demands. Discuss the problems existing in the process of using these new media, make suggestions to optimize the use of Microblog and WeChat for the better satisfaction of the audience's needs. This research will make positive effects on the improvement of the communication efficiency of government new media.
\end{abstract}

\section{Introduction}

According to the "Development Report on China's New Media (2016)", China's microblog certification-account for government affairs (including Sina Microblog and Tencent) reached 289,000, with cumulative coverage of 40 billion people to the end of 2015. In 2015, government affairs microblog reached 21.027 million, with the forwarding and comments amounted to 270 million, the fans increased 17.6\% compared with the year earlier. By the end of 2015, the WeChat public account of government affairs reached 100,000, pushed more than 4.05 million times, with the amount of articles of 30 million and the cumulative reading of more than 2 billion times. In the process of shaping the network-image of the government, the microblog and WeChat has gradually become an important way of information disclosure, government affairs propaganda and network consulting of government affairs. It has also become an important way for people to obtaining government information and discuss public events. Therefore, investigating the usage of Microblog and WeChat for government affairs on the position of users, whether the current development of Microblog and WeChat meet the public expectation, which factor affect the usage and satisfaction of the Microblog and WeChat for the public have theoretically significance and practice value, the answer to these questions can accurately assess the spread effect of Microblog and WeChat, which can give advice for the development of new media for government affairs and the elevation of government network image.

\section{Research methods.}

"Use and gratifications" is an audience-centered theory, which argues that people are actively looking for specific media and specific content to achieve certain satisfaction (or results). In 1974, the communication experts Katz etc. summarized the media contact behavior as a "social factor + psychological factor $\rightarrow$ media expectation $\rightarrow$ media contact $\rightarrow$ demand satisfaction" process, put forward the basic pattern of "use and gratifications". In 1977, the Japanese scholar Takeuchi YuLang made a number of additions to this pattern . The driving question of "Use and gratifications" is: Why do people use media and what do they use them for? Did the media meet the needs of the demand? And which demand? All these questions are the core research area of "Use and gratifications" theory.

The combination of qualitative and quantitative methods was used in this paper. Firstly, questionnaires were given out to the users of Microblog and WeChat for government affairs, and 
statistical analysis were made for the study of people's behavior, motivation and satisfaction degree. Then, draw the corresponding conclusions based on the theory of "use and gratifications" and the results of statistical analysis. Finally, put forward the strategies and recommendations based on the conclusions and problems.

The survey was conducted in the form of paper questionnaires and electronic questionnaires. A total of 400 questionnaires were given out from March 16, 2017 to March 26, 2017, and 364 questionnaires were retrieved with a recovery rate of $91 \%$.

\section{Use and gratifications analysis of investigated people}

\subsection{The concern behavior of investigated people}

The sexes of investigated people in the 364 effective questionnaires were $60.06 \%$ and $39.94 \%$ for men and women, respectively. It can be inferred that men are more concerned about the Microblog and WeChat of government affairs than women, this phenomenon may occur due to the gender differences, because men are more interested in public affairs and political content.

Among the respondents, $15.09 \%$ were in the age of $18-25$, $30.40 \%$ were $26-35$ years old, $33.64 \%$ were $36-45$ years old, $17.47 \%$ were $46-55$ years old, and $3.40 \%$ were above 55 years old. It can be concluded that the middle-aged people and youth are more concerned about the Microblog and WeChat for government affairs.

\subsection{The way to access government affairs information}

The survey shows that the main way for people to obtain government affairs information are the government website, WeChat, Microblog, paper documents and government text messages, which are decrease successively. Among these ways, the government website accounted for 37.18\%, Microblog accounted for $28.55 \%$, WeChat accounted for $17.58 \%$, paper documents accounted for $14.52 \%$, government SMS accounted for $2.17 \%$. The data shows people are preferring to government website for government affairs information, then followed by Microblog.

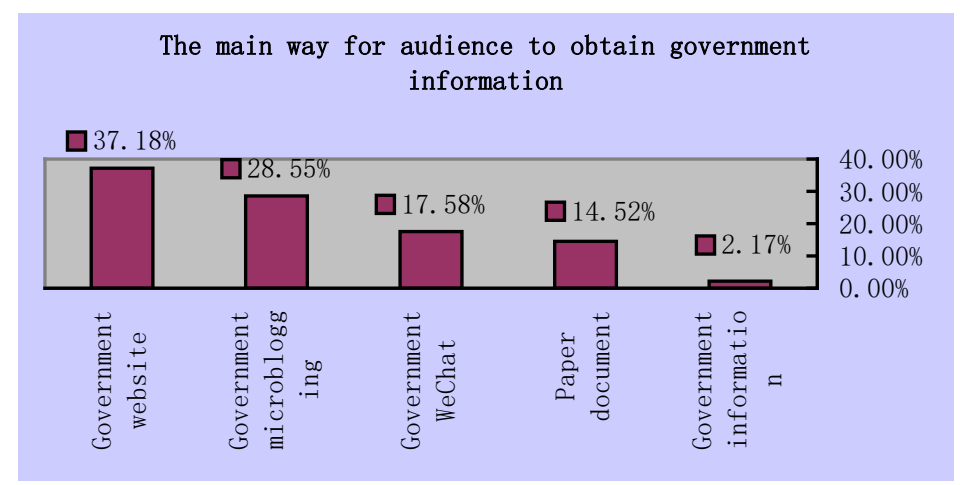

Figure 1. The main way for audience to obtain government information

\subsection{The way to follow government affairs Microblog and WeChat}

In the investigation of the way for public to follow the Microblog and WeChat for local government affairs, find by themselves accounted for $24.12 \%$, tracking the read article accounted for $15.29 \%$, Microblog or WeChat public account recommended accounted for $17.35 \%$, friends recommended accounted for $11.41 \%$, work or study requirements must subscribe accounted for $10.63 \%$, government officially released accounted for $7.31 \%$, occasionally found online account for $7.54 \%$, social networking sites accounted for $4.59 \%$ and reading newspapers and magazines accounted for $1.76 \%$. The result shows more people are initiative pay attention to the new government affairs media, followed by recommendation ways. 


\subsection{The degree of people pay attention on Microblog and WeChat for government affairs}

Among the 364 respondents, 82.81\% of the people will use Microblog, 64.06\% of the people followed the Microblog of government affairs, but the people pay attention to the Microblog of government affairs frequently accounted for only $17.81 \%$, occasionally concerned people accounted for $83.19 \%$. On the other hand, $95.6 \%$ of the people will use WeChat, $64.71 \%$ of the people followed the WeChat of government affairs, the people pay attention to the WeChat of government affairs frequently reached $36.53 \%$, occasionally concerned people accounted for $63.47 \%$. Results shows that more people are using WeChat, and the proportion of people pay attention to government affairs frequently is higher in WeChat than Microblog. This phenomenon is related to the rapid development of WeChat in recent years. For the people using Microblog or WeChat to obtain government information, $73.53 \%$ and $65.62 \%$ of the people show increasing concern frequency on government affairs for WeChat user and Microblog user, respectively.

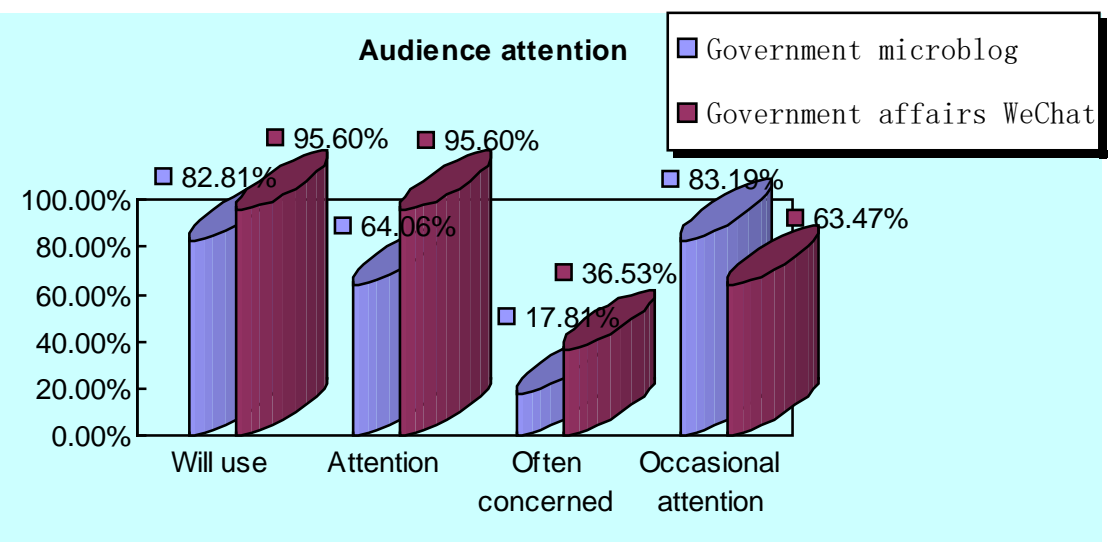

Figure 2. Audience attention

\subsection{The content of people concerning}

In the 364 effective questionnaires, people are mostly pay attention to the current affairs and education from the Microblog information released by government affairs, then in sequence of civil affairs, culture, security, finance, entertainment, tourism and environmental protection. For the WeChat information, people are most concerned about the current affairs, followed by education, culture, civil affairs, tourism, entertainment, finance, security and environmental protection.

\section{The satisfaction status of people's needs}

For the question of "whether the government affairs Microblog and WeChat improved the public awareness on specific incident", $70.97 \%$ of the respondents said improved and $27.42 \%$ did not see any change, only $1.61 \%$ of the respondents think Microblog and WeChat decreased the public awareness. From the statistical results, the degree of satisfaction generally shows a high level, such as government information disclosure, emergency incident information, personal information service and other needs are usually can be met.

For the question of "whether the government affairs Microblog and WeChat improved the degree of public participation in specific incident”, $73.53 \%$ of the respondents deem it increased public participation, $20.59 \%$ of the respondents deem that there is no change, Only $5.88 \%$ of the respondents deem that it reduced the public's participation degree. The result shows, when people's "right to know" is satisfied, the social participation needs have been better met either.

For the question of "whether the information obtained from government affairs Microblog and WeChat is useful”, 52.94\% of the government affairs WeChat user and 40.62\% of the Microblog user think it is useful. By following the local government affairs Beibo and WeChat, $70.59 \%$ of the respondents raised the degree of satisfaction for local government. From the statistical results, the opening and promotion of new government media, satisfied the public information needs to a certain extent, improved the public's satisfaction on the government. 
In the aspect of "the role of Microblog and WeChat on government information disclosure", the respondents believe that the primary function of government new media is making the public more clear on the local government's recent developments and facilitate the local public life, secondly, greatly promoted the government information disclosure, the third function is that the new government media closer the distance between the public and the government, enhance the credibility of the local government, the fourth function is strengthen the political participation of the public, the fifth function is enable government response timely, to ease public sentiment and guide public opinion, the sixth function is social management innovation. The survey shows public basically recognized the new media increased the openness of government information, improved the government work efficiency and the credibility of the government, on the contrary, there is nobody think the government new media negatively affect government affairs.

\section{Existing problems}

According to this survey, we can see the government affairs Microblog and WeChat nicely meet the needs of most of the respondents, and improved the government's media image, but there are still many problems, which mainly shows:

\subsection{Government information disclosure is tardy}

The survey shows the biggest problem on government affairs Microblog and WeChat information disclosure is information tardy, which account for $47.06 \%$ and $56.25 \%$ for Microblog and WeChat users, respectively. For the government affairs Microblog, $7.81 \%$ of the user deem the Microblog update is very timely, $25.00 \%$ of the user deem the update is timely, $46.88 \%$ of the user deem the update is in ordinary level, and the proportion of less timely and serious lag accounted for $15.62 \%$ and $4.69 \%$, respectively; while for the government affairs WeChat, $14.71 \%$ of the user believe the update is very timely, $26.47 \%$ of the user believe the update is timely, $47.06 \%$ of the user deem the update is in ordinary level, and the proportion of less timely and serious lag accounted for $8.82 \%$ and $2.94 \%$, respectively. The result shows although the government affairs WeChat update faster than the Microblog, the overall update speed still can not satisfying the public.

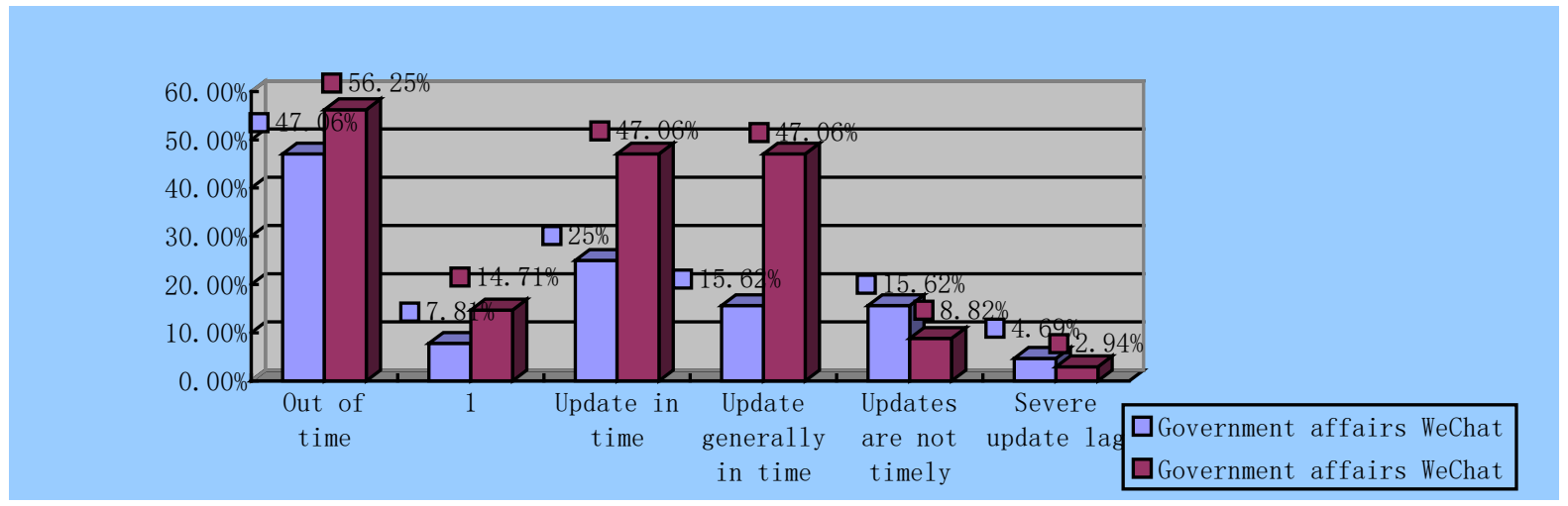

Figure 3. Update speed

\subsection{Government information insufficient disclosure, poor pertinence and lack feedback}

Among the 364 respondents, $44.12 \%$ of the respondents believe that the disclosure information on WeChat can be further improved, while $34.38 \%$ for Microblog. In the targeted, $32.35 \%$ of the respondents believe that the disclosured information on WeChat has a poor pertinence, and can not meet the public demand, while $46.88 \%$ for Microblog. For the feedback time, $41.18 \%$ of the respondents believe the government WeChat did not timely reply the public's consults, and $45.31 \%$ of the respondents believe the government Microblog did not timely reply the public's consults.

\subsection{Government information presented monotonous, accuracy need to be improved}


Among the 364 respondents, $17.65 \%$ of the respondents considered that the information issued by the government affairs WeChat was monotonous, lack of novelty and diversity, while $43.75 \%$ for government affairs Microblog. On the issue of information accuracy, 14.71\% of respondents believe that the accuracy of the information disclosed by the government WeChat needs to be improved, while $29.69 \%$ for government affairs Microblog.

\section{The ways for effective use of the government affairs Microblog and WeChat}

From the basic patterns of "use and gratifications" theory of Katz and Beneguro, it can be seen that the audience has already expect the media to meet their needs before using it. Therefore, the media must take into account the needs of the audience to make a effectively spread result. Meanwhile, the media itself will affect or restrict its usage of the audience.

Based on the problems discussed in this article, we suggest improve the effectiveness of the Microblog and WeChat in three aspects: content production, publishing and media use.

\subsection{Improve the connotation service, make both good utility and administration for content production}

Government information should be closely integrated with the basic life and the audience based on government work, can not only publish the activities of administrator, or the information useless or have none business with people's livelihood. It is not only necessarily to reflect the government work, as much as possible to improve the government information disclosure, but also make some useful and profitable information which fit with the audience needs.

\subsection{Change the concept of communication, to achieve timely, fast and diversified information publish and feedback}

If the government want publish information and achieve publicity function with the new media platform, to maintain social and information stability and leading the right of public opinion, they should follow the regularity of media development, change the concept of development, to initiatively understand the needs of specific audiences, make effective and flexible media management strategy. Government information release and update should be timely and fast, which will seize the dominance of public opinion at the shortest time; the presentation of the information should be diversified, innovative and straightforward to the audiences. For the public consultation, the feedback of the new media platform should timely and make efficient solution, enhance the response speed and public awareness of the incident, meet the diverse needs of the audience.

\subsection{Avoid weaknesses, complementary advantages for Microblog and WeChat}

With the development of information technology, varieties of new electronic media and instant messaging tools have sprung up. The rational use of these "technology bonus" can effectively promote social governance and transformation of government public service, build up bridge between the government and the public to facilitate communication, to achieve "let the people run less for government service, solve the problem of government service of difficult and slow". But these technologies still have some limitations, different technical have different advantages and disadvantages, for the example of Microblog and WeChat, the government affairs Microblog are more open for the published information, while the government affairs WeChat deliver information more accurately, and the information is more diversified. In the process of information disclosure, government departments associate with the responsibility based on their characteristics, take advantages of Microblog and WeChat to complement the weakness.

\section{Acknowledgements}

In this paper, the research was sponsored by the Henan soft science research project (Project No.152400410013) ; Henan provincial high school youth backbone teacher funding scheme project 
(Project No.2015GGJS-276) ; Henan province philosophy and social science planning project (Project No.2017BXW009).

\section{References}

[1] W. Richard, T. Turner, The theory of communication: analysis and application, China renmin university press. 2007, 433-434.

[2] J.P. Xi, Information on http://news.xinhuanet.com/politics/2016-04/19/c_1118672059.htm.

[3] Y.C.Zhang, Using the theory of "use and satisfaction" to analyze the spread of government affairs WeChat, Youth Journalist. 2(2014).

[4] J. Shi, Study on the "double micro linkage" mode of government affairs service-Based on the text analysis of the WeChat of government microblog and government affairs in Shanghai, Electronic Research. (2)2016.

[5] Information on China Internet Network Information Center (CNNIC). The 38th statistical report on the development of China's Internet network 